\title{
2 Sprengungen: Ein LoRd am AleXanderplatZ
}

In einem Land leben, wo es keinen Humor gibt, ist unerträglich, aber noch unerträglicher ist es in einem Land, wo man Humor braucht. ${ }^{1}$ (Bertolt Brecht, 1949)

Indem das Kino uns die Welt erschließt, in der wir leben, fördert es Phänomene zutag, deren Erscheinen im Zeugenstand folgenschwer ist. Es bringt uns Auge in Auge mit Dingen, die wir fürchten. Und es nötigt uns oft, die realen Ereignisse, die es zeigt, mit den Ideen zu konfrontieren, die wir uns von ihnen gemacht haben. ${ }^{2}$ (Siegfried Kracauer, 1960)

Familien sind stets Elemente von Gruppen; und diese sind [...] in gemeinsamen Atmosphären begründet. [...] Im Zeichen des Atmosphärischen laufen [...] zahlreiche unsichtbare, gleichwohl trennscharfe und wirksame Grenzen durch die ganze menschliche Lebenswelt. ${ }^{3}$ (Hubert Tellenbach, 1968)

Dem ,leichten' Genre angemessen, kommt die Geschichte, die EIN LORD AM AlEXANDERPLATz (1967) erzählt, recht harmlos daher. Ewald Honig (Erwin Geschonneck), ein schon etwas betagter Lebemann, der im Westen wegen betrügerischer Eheversprechen in Konflikt mit dem Gesetz geraten ist, siedelt nach verbüßter Strafe von München nach Ostberlin über. Dort lebt seine erwachsene Tochter Ina (Angelica Domröse), der er von nun an ein guter Vater sein will. Mit seinem alten Mercedes 300 fährt er in die Hauptstadt der DDR ein, findet sich jedoch alsbald durch verschiedene weibliche Zufallsbekanntschaften erneut in überwunden geglaubte Verwicklungen verstrickt.

Gleich drei Damen mittleren Alters erliegen seiner eleganten Erscheinung, seinem Charme und der unwiderstehlichen Anziehungskraft, die das hinter der Nobelkarosse vermutete „Auslandsvermögen“ auf diejenigen ausübt, die sich in Zeiten gesellschaftlicher Neuorientierung nach einem potenten Versorger sehnen: Da ist zum einen die Leiterin einer Kfz-Werkstatt (Erika Dunkelmann), wohin Honig gleich nach seiner Ankunft in Berlin die altersschwachen Bremsen seines Mercedes führen. Da ist zum anderen eine frisch gebackene Witwe (Carola Braunbock), die mit etwas Vermögen und einem durch den Tod des Gatten überflüssig gewordenen Wartburg-Kombi seit kurzem allein im Leben steht. Da ist drittens eine gewisse Frau Müller (Marianne Wünscher), die dem Kosmetiksalon

1 Bertolt Brecht: Flüchtlingsgespräche [1949]. In: Ders.: Gesammelte Werke, Bd. 6 (Prosa 2). Frankfurt am Main 1967, S. 1459.

2 Siegfried Kracauer: Theorie des Films. Die Errettung der äußeren Wirklichkeit [1960]. In: Ders.: Werke, Bd. 3, hg.v. Inka Mülder-Bach u. Mitarbeit v. Sabine Biebl. Frankfurt am Main 2005, S. 467. 3 Hubert Tellenbach: Das Atmosphärische als das Umgreifende [1968]. In: Stephan Günzel (Hg.): Texte zur Theorie des Raums. Stuttgart 2013, S. 64-66, Zitat S. $64 \mathrm{f}$.

Ә OpenAccess. (c) 2020 Michael Wedel, publiziert von De Gruyter. (cc))BY-NC-ND Dieses Werk ist lizenziert unter der Creative Commons Attribution-NonCommercial-NoDerivatives 4.0 License. 
vorsteht, in dem Honigs Tochter angestellt ist, und mit der er am Ende sehr zum Verdruss der anderen beiden tatsächlich die Ehe eingehen wird.

Durch die unverhoffte Rückkehr des Vaters schlägt auch das Leben seiner Tochter, zumindest vorübergehend, eine neue Richtung ein. Unter dem Eindruck des vermeintlich vom Vater importierten gehobenen Lebensstandards verabschiedet sich Ina von Wolfgang (Jürgen Reuter), ihrem jugendlich ungestümen Verehrer aus dem Ruderverein, um sich reiferen Männern zuzuwenden, die ihr hübsche Kleider, ein Motorboot oder eine Reise zum Plattensee bieten können. Es dauert nicht lange, bis die Volkspolizei auf die verdächtigen Umtriebe von Vater und Tochter aufmerksam wird - nicht zuletzt wegen der von ihnen separat geschalteten Kontaktanzeigen. Die Ermittlungen werden allerdings eher halbherzig vorangetrieben. Polizeihauptmann Pahl (Friedo Solter) setzt die gerade zu Fortbildungszwecken in der DDR weilende ungarische Kollegin Johanna Farkas (Monika Gabriel) und den Kriminalpsychologen Dr. Achim Engelhardt (Armin Mueller-Stahl), Autor eines Standardwerks zur „Täterpsychologie“, auf die „Fälle“ an. Nachdem Honig verheiratet, Ina zu Wolfgang zurückgekehrt und aus den beiden Ermittlern, die sich zwischendurch gegenseitig für die Gesuchten gehalten hatten, ein drittes Paar geworden ist, löst sich zu guter Letzt vor Gericht alles in jenes Wohlgefallen auf, auf das es Günter Reischs Komödie von Anfang an abgesehen $\mathrm{zu}$ haben schien.

EIN LORD AM ALEXANDERPLATZ wäre nicht mehr als eine amüsante Kapriole im Werk Reischs und bliebe eine Fußnote in der Gesamtproduktion der DEFA, nähme man den Film lediglich zum Anlass, den Einfallsreichtum hervorzuheben, mit dem seine Handlung erdacht und von Regisseur und Darstellern ins Werk gesetzt wurde, um damit den schlichten Nachweis $\mathrm{zu}$ führen, dass auch bei der DEFA funktionierende und heute noch unterhaltend anzuschauende Genrefilme möglich waren. Im Rückblick erscheint EIN LORD AM ALEXANDERPLATZ weit aufschlussreicher und auch weitaus prekärer in der Geschichte der DEFA zu stehen, als es sein Komödien-Inhalt für sich genommen vermuten lässt. Und das in mehrfacher Hinsicht: Mit Blick auf die kulturpolitischen Rahmenbedingungen, unter denen er entstanden ist; mit Blick auf die Genrepoetik, die er in der konkreten historischen Situation, in die er eingelassen ist, verfolgt; sowie mit Blick auf die ästhetischen Formen und gesellschaftlichen Diskurse, die er in Verbindung mit seinem Sujet ins Spiel bringt. Von allen drei Seiten her sollen im Folgenden Schlaglichter auf den Film geworfen werden. Am Ende laufen meine Überlegungen und Beobachtungen auf die These hinaus, dass EIN LORD AM ALEXANDERPLATZ im Spannungsfeld der von Brecht bezeichneten gesellschaftlichen Aporie und im Bereich der atmosphärischen Auskleidung der Handlung eine Reihe von Strategien entwickelt, die - mit Kracauer gesprochen - auf humorvolle 
Weise die Dingwelt der Großstadt ins Spiel bringen, um die Ideen zu prüfen, die man sich von ihr macht.

\section{Romantik und Realität}

Jede noch zu schreibende Geschichte der Filmkomik in der DDR hätte Günter Reisch einen der vordersten Plätze einzuräumen. Kein zweiter Spielfilm-Regisseur der DEFA hat die Genres der Komödie und des Lustspiels mit vergleichbarer Akribie und Ausdauer bedient, niemand sonst mit ähnlicher Insistenz über politische Klimaveränderungen und die Gezeiten des Geschmackswandels hinweg die spielerischen Freiheiten des Komischen immer wieder aufs Neue erprobt, ihre gesellschaftliche Relevanz ausgetestet. Nicht weniger als dem zweiten Zentralstrang seines Filmschaffens, den zumeist im Duktus einer ernsthaft aufzuarbeitenden Genealogie gehaltenen Heldenporträts aus der Geschichte der sozialistischen Bewegung, hat Reisch seinen Komödien die vielfach attestierten Stärken seiner Regie in den Dienst gestellt: das Gespür für Schauspieler-Inszenierung und präzise Figurencharakterisierung, den Instinkt für passende formale Zuspitzungen durch avancierte Bild-Ton-Montagen, vor allem aber die durch wachsende Routine sukzessiv verfeinerte Gabe, eine Szene in atmosphärischer Verdichtung auf den Punkt zu bringen. ${ }^{4}$

Den über Jahrzehnte durchgehaltenen Wechsel zwischen beiden Hauptsträngen seines Schaffens wollte der Regisseur dabei weder als logische Gesetzmäßigkeit noch als reines Zufallsprodukt betrachtet wissen. Vielmehr sprach er einmal von einer „berechtigten Notwendigkeit“, die sich nicht allein aus künstlerischen Erwägungen ableiten ließe, sondern gesellschaftlichen Gegebenheiten und den Bedürfnissen des Publikums Rechnung trug. ${ }^{5}$ Es mag bis auf weitere vergleichende Einzeluntersuchungen dahingestellt sein, ob der von Kritikern an seinen historischen Filmen beobachtete künstlerische „Reifeprozess“ von eher schematisch geprägten Handlungs- und Figurenentwürfen zu einer „psychologisch tiefer lotenden Analyse [...] bis hin zu individualisierten Charakterbildern“ ${ }^{\text {6 }}$ seinem Werk insgesamt zu unterstellen ist. Die Spanne zwischen JungES GEMüsE (DDR 1956) und MAIBowle (DDR 1959) am einen, NELKEN IN AsPIK (DDR 1976)

\footnotetext{
4 Vgl. Lutz Haucke: Günter Reisch. Von der Notwendigkeit des historischen Revolutionsfilms und der Filmkomödie. In: Rolf Richter (Hg.): DEFA-Spielfilmregisseure und ihre Kritiker, Bd. 1. Berlin 1981, S. 125-149, hier S. 133. Vgl. a. Ludmilla Kasjanowa: Günter Reisch. In: Dies. und Anatoli Karawaschkin: Begegnungen mit Regisseuren. Berlin 1974, S. 71.

5 Zit. nach Kasjanowa: Günter Reisch, S. 66.

6 Haucke: Günter Reisch, S. 125.
} 
und ANTON DER ZAUbERER (DDR 1978) am anderen Ende des Zeitpfeils legt eine solche Entwicklung seiner Arbeit im Bereich der Filmkomödie zumindest nahe. Wobei freilich der Hinweis zu berücksichtigen ist, dass man es in diesem Genre im Allgemeinen eher mit „Typen“ zu tun hat, die selten zu „Charakterbildern“ vorstoßen. $^{7}$

Quer zur Werkchronologie sind die historischen und die komischen Sujets bei Reisch über die Konstante eines „inneren Grundthemas“8 ${ }^{\text {“8 }}$ miteinander verbunden, das seinem Filmschaffen epochen- und genreübergreifend Kohärenz verleiht. Diesem „inneren Thema nachzugehen“, sei ihm beim Filmemachen immer am wichtigsten gewesen, gibt Reisch Ende der 1970er Jahre zu Protokoll:

Sie können es in vielen meiner Filme entdecken - in den Auseinandersetzungen mit der romantischen Vorstellung von Revolution und Entwicklung des Sozialismus, in den Schwierigkeiten, sich davon zu lösen, und im notwendigen Übergang zu den disziplinierten Formen des Kampfes und des Zusammenlebens. Und man wird die eigene Disziplinierung darin entdecken, die Einordnung in die Gesellschaft, und daß darin einer seine Freiheit sucht. ${ }^{9}$

Was sich in der hier gewählten Diktion liest, als sei es allein auf die Protagonisten seiner historischen Filme gemünzt, lässt sich an anderer Stelle, wo vom Erkennen des Zusammenhangs „zwischen abenteuerlichen romantischen Vorstellungen und den Realitäten des Lebens“ die Rede ist, auch als im Hintergrund wirksames Leitprinzip der Komik seiner Lustspiele auslegen. ${ }^{10}$ Als solches lässt es sich nicht zuletzt anwenden auf die dramaturgische Folie, auf der ein Film wie EIN LORD AM ALEXANDERPLATz beileibe nicht nur seine Hauptfigur als einen mit überholten ,romantischen“ Vorstellungen behafteten Charakter entwirft, der sich nach seiner Umsiedlung aus dem Westen in der neuen sozialistischen Lebenswirklichkeit zurechtfinden muss. „Mir scheint“, so Reisch,

daß der Lustspielfilm seine Gestalten in eine zum komischen Widerspruch herausfordernde Situation versetzt. Sie sollte originell und unverwechselbar sein. Erwin Geschonneck als Lord am Alexanderplatz, erfahrener Heiratsschwindler in der bürgerlichen Gesellschaft, kommt in die DDR und will sein Leben in ehrlicher Ruhe beenden. Aber einige Frauen hier,

7 Günter Reisch: Bist Du ein Arbeiter, oder bist Du kein Arbeiter? In: Ders.: Anspruch, Realisierung und Zuschauer. Ausgewähltes - 70er Jahre. Eine Dokumentation. Sonderheft Aus Theorie und Praxis des Films 7/8, 1980, S. 76.

8 Günter Reisch: In schwierigsten Situationen immer optimistisch. In: Ostsee-Zeitung (16.09. 1978); zit. nach Reisch: Anspruch, Realisierung und Zuschauer, S. 90.

9 Günter Reisch: Erinnerungen im 30. Jahr der DDR (Teil I). Ein Werkstattgespräch - Diskussionspartner Lutz Haucke. In: Filmwissenschaftliche Beiträge 1, 1979, S. 5-34, hier S. 6.

10 Reisch: In schwierigsten Situationen immer optimistisch, S. 90. 
ungewohnt solcher Artigkeiten wie Rosenschenken, Handküsse und anderer Aufmerksamkeiten außerhalb des Frauentages, stürzen sich auf diesen charmanten Kollegen und bringen ihn in die gefährlichsten Versuchungen. Das sind Ansätze für ein Lustspiel. ${ }^{11}$

\section{Vergangenheit und Gegenwart}

Reisch und sein Co-Autor Kurt Belicke entwickeln diese Ansätze in ihrem Film, der zum ersten Produktionsjahrgang nach dem verheerenden 11. Plenum gehört, ${ }^{12}$ in dessen Folge rund ein Dutzend Spielfilme der Zensur zum Opfer fielen, nicht mehr im ideologischen Sinne einer rein kontrastiven Satire. Deren Wiederbelebung war im Anschluss an den im Oktober 1952 abgehaltenen 19. Parteitag der KPdSU als Mittel des Klassenkampfes gefordert und in den 1950er Jahren in der Sowjetunion wie bei der DEFA im Film rege praktiziert worden. Anstelle die sich im Denken und Verhalten der Protagonisten manifestierenden „Überbleibsel“ des kapitalistischen und bürgerlichen Zeitalters zum Gegenstand eines „zornigen, geißelnden“ Verlachens zu machen, mit dem sich die (sozialistische) Gegenwart von der (bürgerlich-kapitalistischen) Vergangenheit vermeintlich auf einen Schlag befreit, wählt EIN LORD AM ALEXANDERPLATz die feinere Klinge der humorvollen Beobachtung seiner Helden und einen Umgang mit den in den sozialistischen Alltag mitgeschleppten Schwächen, der von subtilen Ironien durchzogen ist. ${ }^{13}$

11 Das Lustspiel erlaubt, das Leben spielerisch und vorwiegend heiter zu betrachten. In: Kino DDR. Progress-Pressebulletin 9, 1978; zit. nach Reisch: Anspruch, Realisierung und Zuschauer, S. 95.

12 EIn LORd Am AleXANDERPlatz, der seine Premiere am 3. März 1967 hatte, gehört neben Horst Seemanns Debüt HochzeITSNaCht IM REgen (UA: 15. Mai), Wolfgang Luderers MeIne Freundin Sybille (UA: 25. Juni) und Ralf Kirstens Frau Venus UND Ihr Teufel (UA: 1. Juli) zu den vier Spielfilmkomödien, die von der DEFA 1967 in die Kinos gebracht wurden. Sie machen damit mehr als ein Drittel der insgesamt elf Spielfilmpremieren dieses Jahres aus (Kinderfilme nicht mitgezählt). Deutlich wird in ihnen, dem Urteil Klaus Wischnewskis zufolge, „der generelle Versuch der DEFA, mit leichter Kost Gegenwart freundlich zu umspielen, konventionell zu verschönen oder ihr in undeutliche Historie auszuweichen“. Klaus Wischnewski: Träumer und gewöhnliche Leute. 1966 bis 1979. In: Ralf Schenk (Red.): Das zweite Leben der Filmstadt Babelsberg. DEFA-Spielfilme 1946-1992. Berlin 1994, S. 213-263, hier S. 216.

13 Zur ideologischen Mobilisierung der Satire Anfang der 1950er Jahre sowie zur Unterscheidung der verschiedenen satirischen Spielarten vgl. Juri Borew: Der Waffen liebste Gattung. In: Über die Satire im Film, hg. v. Ministerium für Kultur, Hauptverwaltung Film (Beiträge zu Fragen der Filmkunst, Heft 6). Berlin o. J. [1954], S. 7-32 (dort auch die im vorhergehenden Satz gemachten Zitate auf S. 9 und 20). Reisch selbst erinnert sich, dass vorangegangene „Versuche im Genre der Filmsatire“ bei der DEFA wie Wozzeck (D 1947, Georg C. Klaren) oder Die SELTSAMEn ABEnTEuer 
Die beiden zentralen Protagonisten des Films, Honig und seine Tochter Ina, reihen sich auf diese Weise ein in eine Galerie nominell ,negativer Helden', die Reisch dennoch sympathisch wirken lässt. Für sie gilt wie für den egozentrischen Funktionär Amman (Herbert Richter) aus JungEs GEMüsE, den Gerechtigkeitsfanatiker Thomas (Arno Wyzniewski) aus ACH, DU FRÖHLICHE... (DDR 1962) oder den Titelhelden von ANTON DER ZAUBERER, dass sie zwar dem sozialistischen Gesellschaftsideal fremde Vorstellungen und Verhaltensweisen an den Tag legen, unter der kritikwürdigen Oberfläche im Grunde jedoch ein „guter Kern“ steckt, der „sich über belachenswerte Verwicklungen und Verkehrungen schließlich durchsetzt“ und in eine verlässliche „Bekehrung des Helden“ mündet: „Auf die bissigen Attacken wird verzichtet. Reisch setzt auf gutmütigen Humor, auf Spaß, Augenzwinkern, auf das Ausspielen der kleinen Schwächen zu großen Wirkungen. “14

In seiner Handhabung des satirischen Elements geht es Reisch nicht darum, sich der Residuen der Vergangenheit mit den Mitteln der ideologisch motivierten radikalen Überzeichnung schlicht und einfach $\mathrm{zu}$ entledigen. Viel eher geht es ihm um eine auf die Gegenwart gerichtete „Kritik an bestimmten zeitgenössischen Zuständen“, ${ }^{15}$ die über das Lachen den Weg zu einer Form der gesellschaftlichen Selbstkritik freimachen will, die weder dogmatisch und verordnet erscheint noch

DES FRIDOLIN B. (D 1948, Wolfgang Staudte) im Zuge der Formalismus-Debatte „damals von der Kritik ziemlich niedergeknüppelt“ worden seien (Reisch: Erinnerungen im 30. Jahr der DDR [Teil I], S. 11). Eine Wende signalisierte dann der Erfolg von Staudtes DER UNTERTAN (DDR 1951). Sowohl das 1953/54 unrealisiert gebliebene Filmprojekt „Shakespeare dringend gesucht“ nach dem Theaterstück von Heinar Kipphardt als auch Reischs Spielfilmregiedebüt JUNGES GEMÜSE, eine aktualisierende Reprise von Gogols „Revisor“, in dem ein Schriftsteller auf der Suche nach einem literarischen Stoff aus dem Landleben der DDR im betreffenden Dorf irrtümlich für einen Kontrolleur des Ministeriums gehalten wird, sind im unmittelbaren Zusammenhang mit dieser Entwicklung zu sehen. Demgegenüber gilt ACH, DU FRöHLICHE... (1962) Filmhistorikern heute als Vorläufer der realistisch-kritischen Gegenwartsfilme von 1965/66, die auf dem 11. Plenum verboten wurden. Vgl. Michael Grisko: Junges Gemüse (1956) oder „Marxismus“ in Kappeshausen. Günter Reischs Debütfilm im Kontext der zeitgenössischen Lustspiel- und Satiredebatte; ders.: Günter Reischs Weihnachtsfilm ACH, DU FRÖHLICHE... (1962), oder das „Leben ist eine Leberwurst“. Beide in: Michael Grisko (Hg.): Zwischen Historienfilm und Gegenwartskomödie. Studien zum Werk des DEFA-Regisseurs Günter Reisch. Marburg 2012, S. 47-69 und 123-140. Zu den schwierigen Anfängen des auf die inneren Befindlichkeiten der SBZ und DDR gerichteten satirischen Genres vgl. medienübergreifend Sylvia Klötzer: Satire und Macht. Film, Zeitung, Kabarett in der DDR. Köln, Weimar und Wien 2006.

14 Haucke: Günter Reisch, S. 140. Ähnlich wurde die Behandlung der Honig-Figur noch knapp zwei Jahrzehnte später bewertet: „Reisch verspottet diese Haltung nicht, sondern näherte sich ihr mit augenzwinkerndem Humor." Ralf Schenk: Zwischen Komik und Pathos. Der Regisseur Günter Reisch - Skizzen zu einem Porträt. In: Film-Dienst 25, 1997, S. 4-7, hier S. 5.

15 Haucke: Günter Reisch, S. 141. 
die spezifische Gemengelage der „Gleichzeitigkeit des Ungleichzeitigen“ (Ernst Bloch) verkennt. ${ }^{16}$

Reischs in vielen Texten und Gesprächen ausgebreitete ,Sozio-Poetik` der Filmkomödie, die zugleich eine politische Wirkungsästhetik des Genres formuliert, ${ }^{17}$ enthält zahlreiche Hinweise auf eine solche Auffassung. So wolle er ,mit Mitteln der Komödie die Widersprüche in unserer Gesellschaft aufs Korn nehmen, sie mit dem Geschichtsoptimismus, der in mir steckt, heiter lösen helfen oder die generelle Lösbarkeit von Widersprüchen und Konflikten in unserer Gesellschaft andeuten“.18 „Das Lachen auf vielen Ebenen“ solle dabei jeden einzelnen im Publikum zum „dialektischen Denken“ zwingen:

Von der Kunst wird erwartet, daß sie auf Entdeckungen ausgeht. Ich meine in diesem $\mathrm{Zu}$ sammenhang, daß man auch etwas über die komische Seite entdecken kann. Und wenn das Lachen über eine Sache dazu beitragen kann, Überholtes abzuwerfen, dann hat auch das Lachen einen gesellschaftlichen Wert. [...] Das Überholte ist ja nicht Restprodukt einer alten Gesellschaft, auch eine neue, aufstrebende, sich entwickelnde Gesellschaft schafft ja ständig etwas, was sie hinter sich zurückläßt und zurücklassen muß, wenn Dinge zur Konvention zu werden drohen. ${ }^{19}$

Der praktischen Umsetzung dieses Programms hat immer wieder entgegengestanden, dass sich bei der DEFA, wie Reisch wiederholt anmerkt, innerhalb der Komödienproduktion niemals wirklich eine Tradition herausgebildet hat. Durch das Fehlen kontinuierlicher Arbeitsmöglichkeiten in diesem Genre habe sich auch die notwendige Professionalisierung im Umgang mit typischen Stilmitteln nicht

16 Dies führte in der Rezeption des Films mitunter zu ideologischen Irritationen. So heißt es in einer unveröffentlichten Analyse des Films: „Da der Regisseur Günter Reisch für die Gegenüberstellung der zwei Welten, die hier letztendlich aufeinanderprallen müßten, auch auf unserer Seite zum Teil sehr überlebte Handlungsvertreter und Handlungsumgebungen wählt, bleibt die Konsequenz etwas im Unklaren. Dadurch wird die Eindeutigkeit der Aussage oder besser: des Ideengehaltes erschwert.“ (Barbara Demmler: Versuch einer Filmanalyse. „Ein Lord vom Alexanderplatz“, 7-seitiges, undatiertes Typoskript, S. 3f., Nachlass Günter Reisch, Filmmuseum Potsdam, Sammlungen.)

17 So sei prinzipiell der „Unterhaltungswert komischer Gestaltungsmittel [...] nicht zu trennen von gesellschaftlicher Wirkung“ und das Komische deshalb ein „wesentlicher Bestandteil“ seines „ästhetischen Programms“, weil es „ein wichtiges Instrumentarium“ darstelle, „um weltanschauliche Grundhaltungen auszudrücken und festigen zu helfen“. Das Gespräch geht weiter. (Der Film - Abbild oder Entwurf? In: Film + Fernsehen 3 [1977]; zit. nach Reisch: Anspruch, Realisierung und Zuschauer, S. 137.)

18 Günter Reisch: Erinnerungen im 30. Jahr der DDR (Teil II), S. 101.

19 Heiterkeit auf der Waagschale. In: Thüringische Landeszeitung (30.09.1976); zit. nach Reisch: Anspruch, Realisierung und Zuschauer, S. 76. 
einstellen können. Dieser Umstand wiegt umso schwerer, als nach eigener Aussage die „scheinbar leichte Erzählweise, das lockere Arrangement, die Pointierung durch die Montage, die gewitzte Dialogführung, der richtige Musikeinsatz“ den ,inneren Rhythmus“ eines Regisseurs nirgendwo mehr fordere als „im heiteren Genre“. ${ }^{20}$ Eine Ersatzfunktion erfüllte für Reisch zumindest ansatzweise die Produktionsgruppe „Johannisthal“:

Die hatte als einzige ein fest umrissenes Profil, sie war ernsthaft entschlossen, nur das heitere Genre zuzulassen. [...] Das war die Heimat dafür. Dort machten wir den DIEB von SAN Marengo (1963) und EIn LoRd Am AleXANDERPlatz (1967). [...] Das Stacheltier war eine Schule für Regisseure, der Autoren und der Schauspieler für dieses Genre, also auch eine einfach notwendige handwerkliche Basis ... ${ }^{21}$

\section{Politik und Produktion}

Die Kontinuität des unmittelbaren Arbeitszusammenhangs war um die Jahreswende 1965/66 aber auch von politischer Seite her ganz konkret gefährdet. Ein Exposé und die erste Fassung des Drehbuchs, die noch den Titel „Ein Lord vom Friedrichshain“ trug, wurden von Reisch und Belicke noch vor dem 11. Plenum entwickelt, das Mitte Dezember 1965 tagte, eine zweite Drehbuchfassung wurde im Januar 1966 in ministeriellem Auftrag intern begutachtet. ${ }^{22}$ Vor dem Hintergrund der jüngsten Verbote gegenwartskritischer DEFA-Filme wird die wichtigste Feststellung in der Einschätzung des Gutachtens bereits im ersten Satz getroffen: „Dieses Lustspiel hat kein großes gesellschaftliches Anliegen.“23 Ausführlich werden in der Folge die verschiedenen Stufen der Stoffentwicklung rekapituliert, die auf signifikante Umwertungen in der Figurenkonzeption deuten:

Am Anfang gab es ein Exposé, das ausschließlich auf die Verwechslungsgeschichte zwischen den beiden Kriminalisten baute. Der Heiratsschwindler, also der kriminelle Faktor, spielte vorher eine untergeordnete Rolle, er diente aber nur der genannten Verwechslungsmöglichkeit. In der Entwicklung des Stoffes traten die beiden Heiratsschwindler, Honig und dessen Tochter Ina, immer mehr in den Vordergrund. Durch den Konflikt Honigs mit der Gesellschaft bekam der Stoff einen sozialen Aspekt und gewann dadurch an Substanz. [...] Gegenüber der 1. Drehbuchfassung gibt es eine 2. konzeptionelle Verbesserung, die von

20 Das Gespräch geht weiter; zit. nach Reisch: Anspruch, Realisierung und Zuschauer, S. 137.

21 Reisch: Erinnerungen im 30. Jahr der DDR (Teil I), S. 21.

22 Zum 11. Plenum vgl. Andreas Kötzing und Ralf Schenk (Hg.): Verbotene Utopie. Die SED, die DEFA und das 11. Plenum. Berlin 2015.

23 Einschätzung zu dem Drehbuch „Ein Lord vom Friedrichshain“, Johannisthal, 29.01.1966 (Gericke/Hannemann), Nachlass Günter Reisch, Filmmuseum Potsdam, Sammlungen. 
Bedeutung ist. Honig ist nicht mehr der vorsätzliche, aktive Heiratsschwindler. Fast ohne sein Zutun gerät er in die für ihn verfänglichen Situationen, wird seine Vergangenheit erneut heraufbeschworen. Aus Westdeutschland übergesiedelt, hat Honig ursprünglich die feste Absicht, nicht rückfällig zu werden. Zu seinem Unglück begegnet er aber verschiedenen Frauen, die ihn, gefangen von seinen ausgesuchten Umgangsformen, geradezu dazu zwingen, erneut zum Heiratsschwindler zu werden. Diese Konzeption des passiven Rückfalls macht die Anlage der Rolle Honig von vornherein komischer. Gleichzeitig wird durch die größere ironische Distanz zu Honig die Aussage unterstrichen: Es handelt sich bei diesem Heiratsschwindler um eine gesellschaftliche Ausnahme, eine schon überholte Erscheinung. ${ }^{24}$

Auf der Grundlage der im Gutachten erteilten Ratschläge entstand innerhalb weniger Wochen eine dritte Drehbuchfassung, in der die Handlung an verschiedenen Stellen deutlich gestrafft ist und auch die Schauplätze nun geografisch auf den Alexanderplatz verdichtet sind. Folgerichtig trägt diese Fassung erstmals den Titel des späteren Films. Bis Mitte März wurden weitere Stellungnahmen zu dieser neuen Version vorgelegt. Ende April 1966 erfolgten dann die Abschlussbesprechung und die Freigabe. Wie dem internen Schriftwechsel zu entnehmen ist, geschah dies gegenüber dem zunächst avisierten Zeitplan mit einiger Verspätung. ${ }^{25}$ Sie erklärt sich nicht zuletzt dadurch, dass die Prüfung eingereichter Filmprojekte unmittelbar nach dem 11. Plenum äußerst penibel vorgenommen wurde, selbst wenn es sich bei ihnen um vermeintlich ,harmlose“ Lustspiele handelte. Die Verzögerung der Freigabe vom Ministerium hatte zur Folge, dass Drehvorbereitungen, etwa Motivbesichtigungen durch den Szenenbildner Alfred Thomalla, nicht wie geplant durchgeführt werden konnten. ${ }^{26}$

Bis Mitte Juni arbeiteten Reisch und Belicke ein Regiedrehbuch aus, in dem sie nochmals Streichungen einzelner Szenen sowie zahlreiche Umformulierungen im Dialog vornahmen. Da der fertige Film schon in früheren Drehbuchfassungen auf eine Länge von 2.700 Metern projektiert war, wurde nur weniges noch hinzugefügt. Darunter jener Gag, mit dem die Begrüßung der ungarischen Polizistin durch Hauptmann Pahl im Polizeipräsidium endet und der den Bogen schlägt zu den Bildern einstürzender Altbauten in der Eröffnungssequenz des Films: „Am Ende der Szene kippt Pahl den Schnaps aus dem Fenster. Ungeheure Explosion.

24 Einschätzung zu dem Drehbuch „Ein Lord vom Friedrichshain“. Im Zuge dieser Änderungen war vermutlich auch der ursprüngliche Arbeitstitel des Projekts, das zunächst unter „Der Heiratsschwindler" figurierte, fallengelassen worden.

25 Stellungnahme des Ministeriums des Innern zu unserem Drehbuch „Ein Lord vom Alexanderplatz“ (Der Heiratsschwindler), Berlin, 09.03.1966 (Klein), Nachlass Günter Reisch, Filmmuseum Potsdam, Sammlungen.

26 Stellungnahme des Ministeriums des Innern zu unserem Drehbuch „Ein Lord vom Alexanderplatz“. 
Das alte Dia-Gebäude fällt zusammen. Johanna erschrocken: ,Was war das?` Pahl: ,Der Alexanderplatz: ,wir renovieren! ““27 Wie handschriftliche Anmerkungen Reischs im Regiedrehbuch belegen, mussten kleinere Unstimmigkeiten in der Anlage von Nebenfiguren und der Erzählfunktion von Requisiten sowie in der psychologischen Motivation und dramaturgischen Konsequenz einzelner Handlungssituationen noch kurz vor Drehbeginn bzw. während der laufenden Dreharbeiten ausgebessert werden. ${ }^{28}$

Drei Wochen vor der ersten Klappe erhöhte die DEFA-Leitung den Druck auf die Produktionsgruppe. Sie wies Reisch unmissverständlich auf die besondere Situation hin, in der sich das Spielfilmstudio befand:

\begin{abstract}
Werter Genosse Reisch! In unserer letzten Besprechung über das Drehbuch machten wir darauf aufmerksam, daß das Negativ für den Film EIN LORD AM ALEXANDERPLATZ spätestens bis 31.12.1966 an das Zentrale Kopierwerk abgeliefert werden muß. Wie bekannt, hat das Studio durch die aus dem Jahre 1965 abgelehnten Filme nicht nur finanzielle Verluste erlitten, sondern auch im Jahre 1966 ergeben sich durch den verspäteten Produktionsanlauf größere Planrückstände. Die KAG ,Johannisthal‘ ist durch [...] den vom Studio übernommen[en] Plan verpflichtet, diesen Film noch im Jahre 1966 abzuliefern. Andernfalls besteht die Gefahr, daß der Ablieferungsplan und auch der Finanzplan des Studios nicht erfüllt wird. ${ }^{29}$
\end{abstract}

Zur Not müsse der Film eben schon parallel zu den Dreharbeiten geschnitten werden, entsprechende „organisatorische Maßnahmen“ seien zu ergreifen. ${ }^{30}$

Für Reisch stand von vornherein fest, dass Ewald Honig von Erwin Geschonneck gespielt werden sollte, der damit - nach dem Genossen Lörke in Reischs ACH, DU FRÖHLICHE... und der Figur des „Kalle“ Blücher in Frank Beyers KARBID UND SAUERAMPFER (DDR 1963) - die dritte komische Hauptrolle in einem DEFA-Film übernahm. Für die Rolle der Ina Honig wurden zunächst auch Renate von Wangenheim, Jutta Hoffmann sowie die bis dahin noch gänzlich filmunerfahrene Dorit Gäbler in Betracht gezogen. Die Entscheidung für Angelika Domröse, die 1958 von Slatan Dudow für VERWIRRUNG DER LIEBE entdeckt worden war und seitdem in mehreren DEFA-Filmkomödien mitgewirkt hatte, scheint

27 Lord am Alexanderplatz. Ein Filmlustspiel. Regiedrehbuch, DII vom 16.06.1966, Bild 9, Nachlass Günter Reisch, Filmmuseum Potsdam, Sammlungen.

28 Günter Reisch: Handschriftliche Anmerkungen zu einzelnen Einstellungen [undatiert], Nachlass Günter Reisch, Filmmuseum Potsdam, Sammlungen.

29 Schreiben des VEB DEFA-Studio für Spielfilme KAG „Johannisthal“ an Günter Reisch, 23.06. 1966, Nachlass Günter Reisch, Filmmuseum Potsdam, Sammlungen.

30 Schreiben des VEB DEFA-Studio für Spielfilme KAG „Johannisthal“ an Günter Reisch. 
im Rahmen der Drehvorbereitungen erst relativ spät gefallen zu sein. ${ }^{31}$ Ein Coup gelang mit der Besetzung des Kriminalpsychologen durch Armin Mueller-Stahl, der hier erstmals sein Talent für komische Figuren auf der Leinwand zeigen kann.

Den Auftakt der Dreharbeiten zu EIN LORD AM AlexANDERPLATz bildeten Mitte Juli 1966 Aufnahmen im Café „Espresso“ im Lindencorso an der Ecke Friedrichstraße und Unter den Linden. Am zweiten Drehtag wurde in den Karolinenhof gewechselt, wo Szenen auf dem Wasser zur Aufnahme anstanden.32 Gedreht wurde vorwiegend an Originalschauplätzen rund um den Alexanderplatz, laut Drehbuch waren nur sieben Dekorationen im Atelier vorgesehen..$^{33}$ Die von der Studioleitung angestrebte Ablieferung des Negativs bis Ende des Jahres wurde knapp verfehlt. Letzte Dreharbeiten für die Schlussszene im Gerichtssaal fanden noch wenige Tage vor der Pressevorführung Ende Januar 1967 in einem ehemaligen Kino in Köpenick statt, das die DEFA als „Gelegenheitsatelier ${ }^{* 34}$ nutzte.

\section{Schein und Sein}

Die DEFA zielte mit Reischs Komödie ganz dezidiert auf ein jugendliches Publikum, das nach Aussagen von DEFA-Chefdramaturg Günter Schröder zu jener Zeit drei Viertel der Kinobesucher in der DDR ausmachte. ${ }^{35}$ Um das Interesse zu überprüfen, ließ das Studio den Film vor der Pressevorführung „von 70 Jugendlichen verschiedener Berufe“ testen. ${ }^{36}$ Besonderer Wert wurde in diesem Zusammenhang darauf gelegt, dass die musikalische Gestaltung auch für junge Leute ansprechend ausfiel, einzelne Musik-Titel, so der „Puszta-Beat“, wurden parallel zum regulären Kinostart auch auf AMIGA-Schallplatte angeboten. ${ }^{37}$

Die offizielle Premiere von EIN LORD AM ALEXANDERPLATz fand am 3. März 1967, einem Freitag, um 20 Uhr im Berliner Kino „Kosmos“ statt. Das Presseecho fiel überwiegend positiv aus. Durchgehend gelobt wurden die Leistungen der

31 Vgl. Besetzungsliste „Heiratsschwindler“ [undatiert], Nachlass Günter Reisch, Filmmuseum Potsdam, Sammlungen.

32 Vgl. bh: Der Lord vom Alex. In: Berliner Zeitung, 16.7.1966; Ursula Frölich: Der Herr von gestern. In: Wochenpost 38 (14.09.1966), S. 26.

33 Vgl. Frölich: Der Herr von gestern, S. 26.

34 Dieter Borkowski: Kavalier mit Charme und Rose. Demnächst auf der Leinwand: „Der Lord vom Alexanderplatz“. In: Neue Zeit (29.01.1967).

35 [Anon.]: „Lord“ getestet. In: Norddeutsche Zeitung (29.01.1967).

36 [Anon.]: „Lord“ getestet.

37 [Anon.]: „Ein Lord am Alexanderplatz“, Filmwerbung 29, 1967, S. 3. 
Schauspieler, allen voran Erwin Geschonnecks Interpretation des unfreiwilligen Heiratsschwindlers. Geschätzt wurden Regie- und Kameraleistung gerade dort, wo sie unerwartete Akzente zu setzen versucht hatten, etwa in gewagten Schnittfolgen, die gar als Anleihen bei der Kollisionsmontage Eisenstein'scher Prägung wahrgenommen wurden. ${ }^{38}$ Die meisten Kritiker fassten den Film in Begriffe der Satire und beurteilten sein Gelingen im Licht der von dieser Gattung erhofften ideologischen Wirkung. Es entging ihnen dabei keineswegs, dass Reisch in entscheidenden Punkten von der verordneten Formel abwich. Im Film und über ihn hinaus, hielt der Rezensent der Tribüne anlässlich der Premiere von EIN LORD AM ALEXANDERPLATz fest, sei Humor nicht zuletzt deshalb ernst zu nehmen, weil es sicher sei, dass es „noch ideologische Rückstände in den menschlichen Beziehungen gibt“, ebenso wie die Tatsache auf der Hand liege, „dass sie sich unter sozialistischen Lebensverhältnissen ständig verringern“. ${ }^{39}$ Denn wo das Sein Sicherheit gebe, werde durch den Humor der Schein der Lächerlichkeit preisgegeben. Der Kritiker der Neuen Zeit sah sich „nach vielem Gelächter“- ganz der Intention des Regisseurs entsprechend - auch zum Nachdenken angeregt „über manchen kleinen oder auch größeren satirischen Angriff auf alte und neue Kleinbürgereien“. 40

Reisch wurde attestiert, „während der Dreharbeiten einige entscheidende Schritte in das weithin noch unerschlossene Gebiet der humorvollen ,Bewältigung، der Gegenwart“ unternommen zu haben. ${ }^{41}$ Zwar handele es sich bei dieser DEFA-Filmkomödie um eine „recht vergnügliche Geschichte“, weil sich in ihr „echte komische Situationen aus dem Alltag mit einem leisen Hieb auf überholte Lebensauffassungen mancher Kleinbürger verbinden“.42 Dabei spiele sich das Geschehen jedoch „unmittelbar in unserer Gegenwart ab, so daß es nicht nur Spaß macht, zuzusehen, sondern bestimmt auch manchen Zuschauer überlegen läßt, ob der hingehaltene Spiegel nicht auch ihm gelten könnte“ “. ${ }^{43}$

Vereinzelt wurden die klaren Fronten vermisst und die fehlende satirische Bissigkeit vom ideologischen Standpunkt her kritisiert. Nicht verschweigen wollte Rosemarie Rehahn in ihrer Besprechung den Lesern der Wochenpost, dass

38 H.U.: Heiterkeit in Sachen Honig. Das DEFA-Filmlustspiel „Ein Lord am Alexanderplatz“. In: Neue Zeit (08.03.1967).

39 Hermann Schirrmeister: Ein Filmspaß mit Geschonneck. „Ein Lord am Alexanderplatz“ (DEFA). In: Tribüne (07.03.1967).

40 H.U.: Heiterkeit in Sachen Honig. Hervorhebung hinzugefügt.

41 Dieter Borkowski: Der Lord vom Alex. In: Filmspiegel 26, 1966, S. 4-7, hier S. 7.

42 Manfred Beckmann: Heiratsschwindler Honig. „Ein Lord am Alexanderplatz“ - ein humorvoller DEFA-Film, der Vergnügen bereitet. In: Junge Welt (12.03.1967).

43 Beckmann: Heiratsschwindler Honig. 
die Ausgangssituation auf mehr als einen herzhaften Schwank hoffen ließ. Westdeutscher Kleinbürger, umständehalber, doch guter Vorsätze voll, in die DDR übergesiedelt, versucht, sich in der neuen Umgebung zu arrangieren - da steckt eine Zeitkomödie drin, da muß es doch, dachte ich, zu den überraschendsten und vergnüglichsten Kollisionen mit einer Welt kommen, die sich in rund zwanzig Jahren jenseits aller Lebenserfahrungen eines Ewald Honig entwickelt hat. ${ }^{44}$

Als vermeintlicher Schwachpunkt des Films wurde ausgemacht, dass die drei Handlungsstränge - „Honigs amüsante Hochstapeleien, die beachtlich konsequenten Abenteuer der hübschen Ina und die deutsch-ungarischen Kriminalistenbeziehungen“ ${ }^{“ 45}$ - nicht genügend (so die einen) bzw. allzu sehr (so die anderen) miteinander verwoben seien: Sodass entweder die „drei Handlungsstränge ein wenig zu sehr nebeneinander her“46 liefen oder sich die „drei Fäden manchmal allzu sehr“ verwickelten und zu einem „unübersichtlichen Garnknäuel“ anwüchsen, anstelle „ein dramaturgisch ordentliches Gewebe“ zu ergeben. ${ }^{47}$ Eine weitere Schwäche bestand aus Sicht vieler Kritiker in der Länge des Films und einem als rapide empfundenen Spannungsabfall gegen Ende. Die Länge von zwei Stunden sei am Film „der dunkle Punkt“, fand nicht nur Hans Joachim Göring vom Bauernecho, zumindest in der dritten, eigentlich kriminalistischen Handlungslinie hätte es „Möglichkeiten für Kürzungen gegeben“. . $^{8}$ „Im letzten Drittel“ verliere der Film zudem „spürbar an Tempo, die Verzahnung der Honig-Handlung mit der kriminalistischen Suche nach dem Heiratsschwindler gelingt nicht, und auch ein paar Schnitte wären noch zu empfehlen“..$^{49}$ Einem Journalisten sei es „,bei der gegenseitigen Überführungs- und Liebesgeschichte zwischen der ungarischen Kriminalistin [...] und Dr. Engelhardt [...] geradezu langweilig“" geworden. ${ }^{50}$

Der Rezensentin von der westdeutschen Frankfurter Rundschau stach dahingegen vor allem der „modische Appeal“ ins Auge, mit dem der Film sich ausstattet:

44 Rosemarie Rehahn: Er sah aus wie ein Lord... DEFA-Schmunzelfilm von Günter Reisch und Kurt Belicke. In: Wochenpost (17.03.1967).

45 Friedrich Salow: Er sah aus wie ein Lord... In: Filmspiegel 7 (05.04.1967).

46 Schirrmeister: Ein Filmspaß mit Geschonneck.

47 Hans Lücke: Dem Honig auf den Leim gegangen. Heiterer DEFA-Film „Ein Lord am Alexanderplatz“. In: BZ am Abend (07.03.1967); H.U.: Heiterkeit in Sachen Honig. Das DEFA-Filmlustspiel „Ein Lord am Alexanderplatz“. In: Neue Zeit (08.03.1967).

48 Hans Joachim Göring: Gelungener DEFA-Spaß. Großartiger Erwin Geschonneck als „Lord am Alexanderplatz“. In: Bauernecho. Organ der demokratischen Bauernpartei Deutschlands (09.03. 1967).

49 Christoph Funke: Mit Homburg und dem Stern. Unernstes zum Lustspiel „Ein Lord am Alexanderplatz“. In: Der Morgen (Ausgabe B) (05.03.1967).

50 Beckmann: Heiratsschwindler Honig. 
„DDR-Wohlstand wird demonstriert, mit Vorliebe sind Neubauten ins Bild gerückt, man twistet, fährt Wasserski, und eine attraktive Kosmetikerin (Angelica Domröse) interessiert sich zwecks Plattenseereise für nicht ganz unvermögende Herren. “51

\section{Großstadtfilm und Querschnittslogik}

Wie die Zusammenschau erweist, wurde EIN LORD AM AlEXANDERPLATZ von der zeitgenössischen Filmkritik vornehmlich an den dramaturgischen Maßstäben der stringent gebauten Filmkomödie sowie am ideologischen Auftrag der Satire gemessen. Wo der Film von diesen Maßstäben abweicht - mit seiner sympathisierenden Zeichnung der ,negativen' Helden, der exorbitanten Gesamtlaufzeit und dem sich crescendo-artig steigernden Grad der ,burlesken' Handlungsverwirrung -, wurde dies kritisch vermerkt. Keine der Pressestimmen brachte die genannten Eigenschaften in Verbindung damit, dass EIN LORD AM ALEXANDERPLATZ ganz dezidiert noch einen anderen Genre-Subtext bedient, wir es bei ihm nicht zuletzt mit einem Berlin- bzw. Großstadt-Film zu tun haben. ${ }^{52}$ In der Berichterstattung über die Dreharbeiten, die nicht umsonst überwiegend an Originalschauplätzen rund um den Alexanderplatz stattfanden, war dieser zentrale Aspekt des Films noch hervorgehoben worden: „Berlin spielt mit, die Stadt, in der das Neue rascher, augenscheinlicher wächst als die Erkenntnisse des Herrn Honig. "53 Nicht nur die Verpflichtung des Kameramanns Jürgen Brauer, der als ein „Kenner und Könner des Beobachtungsfilms“54 galt und seine Methode auch bei EIN LORD AM ALEXANDERPLATZ anwenden sollte, ebenso der Hinweis, „das Filmteam“ werde „noch manchen Kummer mit dem - sagen wir - wißbegierigen Berlinern haben, die sich vor der Kamera in Positur werfen“, ${ }^{55}$ sind Anhaltspunkte dafür, dass Reisch mit seinem Film der Anschluss an eine bis in die 1920er Jahre zurückreichende Tradition vorschwebte. Schon zum Zeitpunkt der Entstehung

51 Wilfriede Werner: Zwischen Peenemünde und Alexanderplatz. Neue Filme aus Babelsberg. In: Frankfurter Rundschau (25.05.1967).

52 Lediglich in der bereits zitierten unveröffentlichten Analyse des Films wird dieser Bezug hergestellt, wenn es heißt: „Das bewegte Milieu, der Verkehr, die modernen Bauten und vor allem der in einprägsamer Weise verdeutlichte Wiederaufbau kontrastieren mit den Ansichten besonders des Haupthelden, aber auch mit denen einiger anderer Personen, wie z.B. der Witwe.“ Demmler: Versuch einer Filmanalyse, S. 5.

53 Frölich: Der Herr von gestern, S. 26.

54 Frölich: Der Herr von gestern, S. 26.

55 Frölich: Der Herr von gestern, S. 26. 
von EIN LORD AM ALEXANDERPLATZ ging es ihm anscheinend um ,die immer wieder neue Entdeckung der ,Straße‘, des Marktplatzes, der Stätten ungezwungener, uralter und lebenspraller Kommunikation“ in der Fortsetzung der BerlinFilme von Gerhard Klein und Wolfgang Kohlhaase. ${ }^{56}$

Im Sinne von Kracauers These, die „Affinität des Films zum Zufälligen“ zeige sich am deutlichsten in seiner Hinneigung zur „Straße“, vor allem der „Großstadtstraße“ als jenem „Ort, an dem das Zufällige übers Planmäßige siegt und unerwartete Zwischenfälle fast die Regel sind“"57, lässt sich Reischs Komödie damit auch als Versuch betrachten, einen urbanen Raum zu inszenieren, in dem sich zwei Varianten der Modernisierung miteinander ins Spiel bringen lassen: die DDR-Moderne eines genuin sozialistisch geprägten städtischen Lebensraums, lesbar in der Chiffre von der Umgestaltung des Alexanderplatzes zur idealen Kundgebungsstätte im Herzen der Hauptstadt nach dem Vorbild des Roten Platzes in Moskau; ${ }^{58}$ und die Konsummoderne bürgerlichen Besitzdenkens und kapitalistischer Warenzirkulation, die unter dem nur allzu symbolischen Banner des Mercedes-Sterns Einzug hält.

Die Titelsequenz von EIN LORD AM ALEXANDERPLATz montiert diese Versuchsbzw. Versuchungsanordnung zu einer chaotischen Kollision der Kulturen: Sie beginnt mit Bildern vom Verkehrstrubel an der Ecke Friedrichstraße und Unter den Linden, untersetzt mit ironischer Musik und einem Off-Kommentar, der, unter Verweis auf das britische Oberhaus, auf die trügerische politische Macht der Zurschaustellung von Dingen und Verhaltensweisen anspielt. Der „Lord“, der im Mercedes auf die Straße Unter den Linden einbiegt, schlägt, wenn auch unfreiwillig, in der Hauptstadt der DDR ein wie eine Bombe. Dies wird zumindest von der auf die ersten Einstellungen folgenden Parallelmontage suggeriert, die zwischen dem am Steuer einen Schlager singenden Honig und Aufnahmen von einstürzenden Häusern hin- und her wechselt, wobei der alternierend von hinten und vorn gezeigte Neuankömmling beim vermeintlichen Anblick der jeweils am Straßenrand kollabierenden Gebäude sichtlich irritiert zusammenzuckt (Abb. 2.1).

Die Exposition endet damit, dass Honig, einigermaßen verwirrt vom ganzen Drumherum der Gebäudesprengungen und des großstädtischen Verkehrschaos, an eine kreuzende Straßenbahn allzu nah heranfährt und sich mit der Frage einer

56 Auf dem Wege dieses Genres wachsen bei uns nicht die schönsten Bäume. In: Sonntag (23.04. 1978); zit. nach Reisch: Anspruch, Realisierung und Zuschauer, S. 86.

57 Kracauer: Theorie des Films, S. 116.

58 Vgl. Bernhard Kohlenbach: Von der Hauptstadt des Deutschen Kaiserreiches zur Hauptstadt der DDR. In: Landesdenkmalamt Berlin (Hg.): Denkmale in Berlin. Bezirk Mitte, Ortsteil Mitte. Petersberg 2003, S. 82-181, hier S. 174-176. 

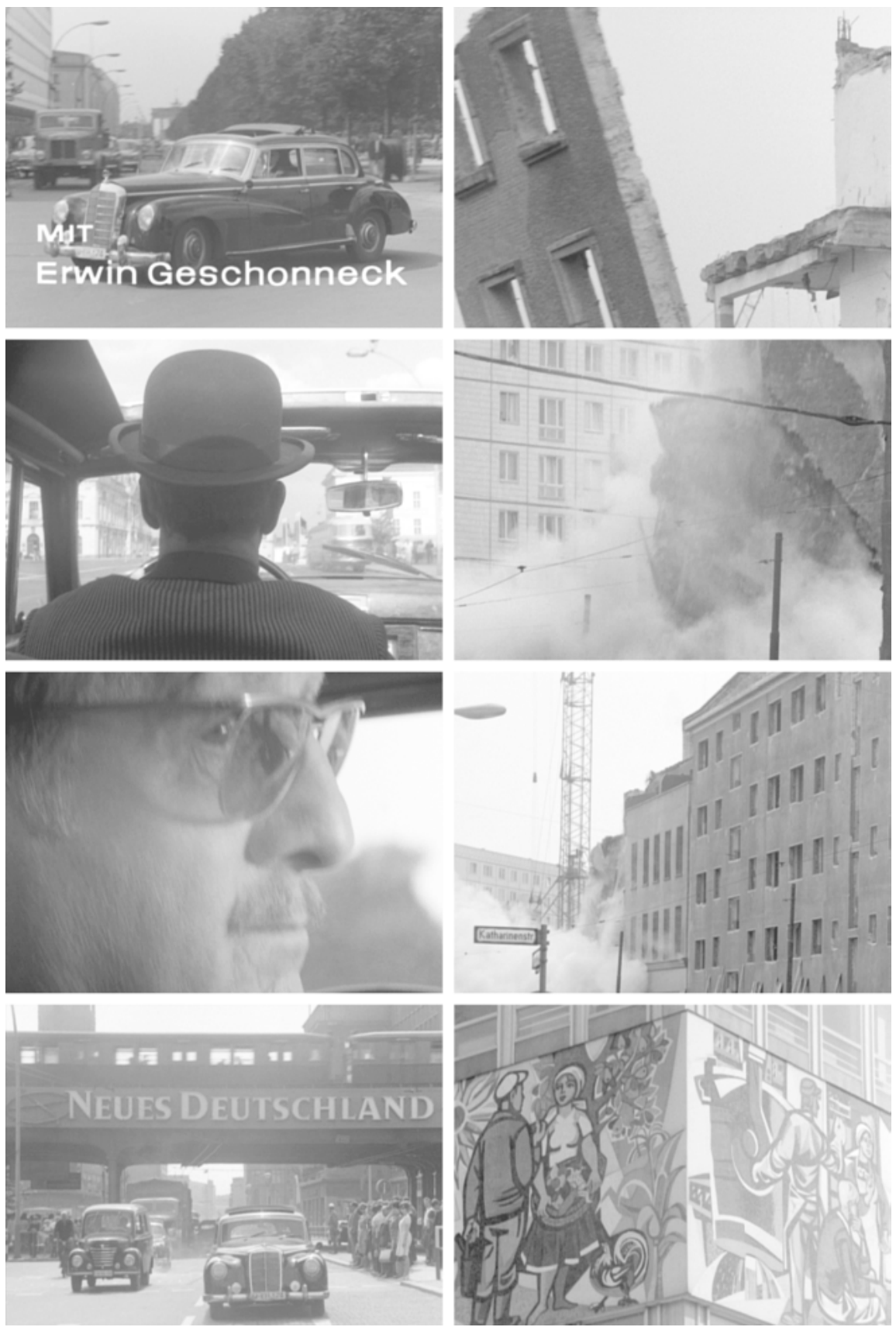

Abb. 2.1: Exposition von EIN LoRd AM Alexanderplatz. 
Volkspolizistin konfrontiert sieht, was denn wohl bei ihm nicht in Ordnung sei, seine Bremsen oder seine Augen.

Konsequent sucht das urbane Setting des Films die klassischen Topoi des Großstadtfilms noch einmal auf, von der Werkstatt zum Kosmetiksalon und in die Zahnarztpraxis, von der Bank zur Polizeiwache, vom Mietshaus übers Treppenhaus in den Hinterhof und immer wieder zurück auf die Straße. Von vereinzelten - seit Menschen AM Sonntag (D 1930, Robert Siodmak, Edgar G. Ulmer und Billy Wilder) und KuHLE WAMPE (D 1932, Slatan Dudow und Bertolt Brecht) obligatorischen - Ausflügen ins Umland abgesehen, bleibt Berlin als aktive Handlungsumgebung stets präsent und wird für Reisch, dem nach eigenem Bekunden auch in seinen Filmkomödien die Atmosphäre im Detail bei der Wahl seiner Sujets wichtiger war als die Setzung dramatischer Höhepunkte, ${ }^{59}$ zum Katalysator für eine Verkettung von „Situationen“, die auch in EIN LORD AM AleXANDERPlatz das „primäre Modul“ seiner Filmkomik darstellen. ${ }^{60}$ Was Reisch aus der Praxis heraus beschreibt, lässt sich als ästhetische Arbeit an der Herstellung von Momenten atmosphärischer Dichte verstehen. Wie von kulturtheoretischer Seite hinzugefügt werden kann, korrespondiert dieser Arbeit an atmosphärischen Räumen und hintergründigen Details eine Form der Wahrnehmung, die weniger von der Verarbeitung narrativer Zusammenhänge geleitet als durch „die Erfahrung der Präsenz von Menschen, Gegenständen und Umgebungen“ bestimmt ist. ${ }^{61}$ Soziologisch lassen sich Atmosphären als „die in der Wahrnehmung realisierte Außenwirkung sozialer Güter und Menschen in ihrer räumlichen (An)Ordnung“ fassen; sie „entstehen durch die Wahrnehmung von Wechselwirkungen zwischen Menschen oder/und aus der Außenwirkung sozialer Güter im Arrangement“.62

An exponierten Stellen wird die großstädtische Rahmung als konkretes Milieu in den Vordergrund zurückgeholt und damit als ultimativer Bedeutungshorizont des Geschehens bewusst gemacht. Etwa wenn unmittelbar vor den Versöhnungs- und Hochzeitsszenen Baukräne ausgiebig von der Kamera abgeschwenkt werden (Abb. 2.2) und der Off-Erzähler auktorial mit den Worten in den Handlungsfortgang eingreift: „Es wird Zeit, dass wir diese verwickelte Geschichte entwirren. Das ist übrigens Berlin von oben, hinter diesen Fenstern geht es zu wie überall ...."63

59 Reisch: Erinnerungen im 30. Jahr der DDR (Teil I), S. 22, 32.

60 Reisch: Erinnerungen im 30. Jahr der DDR (Teil II), S. 109.

61 Gernot Böhme: Atmosphäre. Essays zur neuen Ästhetik. Frankfurt am Main 1995, S. 25.

62 Martina Löw: Raumsoziologie [2001]. Frankfurt am Main 2007, S. 205.

63 EIN LORD AM ALEXANDERPLATZ ist durchsetzt mit optisch, akustisch und verbal ,eingreifenden` Verweisen auf das urbane Milieu, in dem sich die Handlung abspielt. Diese Eingriffe fun- 

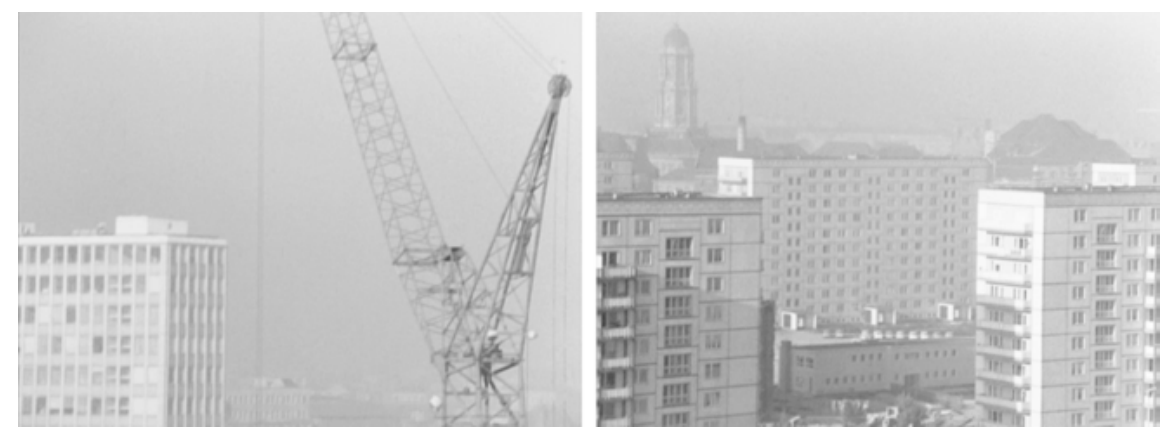

Abb. 2.2: Berlin von oben.

Im Grunde gibt der Film hier selbst die Antwort auf den Vorwurf der fehlenden dramaturgischen Stringenz, den die Presse später gegen das Jonglieren mit parallelen Handlungssträngen und den vielen dazugehörigen Protagonisten erheben wird. Legt der Kommentar doch nahe, dass es sich bei den drei so verwirrend verflochtenen Parallelhandlungen um die mehrgliederige Basis zum Aufbau eines Großstadtpanoramas handelt, das einer Logik des sozialen Querschnitts folgt, wie sie das dem Großstadtfilm immer schon nah verwandte Konzept des ,Querschnittfilms“ kennzeichnet. Seit der Epoche der Neuen Sachlichkeit folgen Querschnittfilme scheinbar zufällig der Zirkulation von Gegenständen im Kreislauf der mechanischen Abläufe einer technisierten Massengesellschaft, um am Ende über Verhaltensanalogien und -differenzen ,objektiven' Einblick in die menschliche Natur und die Gesamtheit einer sozialen Struktur zu verschaffen. ${ }^{64}$ Aus dieser Perspektive erschließen sich die zuweilen vagabundierende Handlungsführung ebenso wie das üppig sprießende Figurentableau von EIN LORD AM ALEXANDERPLATZ als kalkulierte Rückgriffe auf ein Muster, über das sich die Vorstellung von einem sozialen Gefüge vermitteln lässt, das die DDR-Gesellschaft in ihrer noch stets vorhandenen Vertikale vermessen will und horizontal weder an der Grenze zu Westdeutschland noch zu anderen Ostblockstaaten (Ungarn) halt macht.

gieren zugleich als Mittel der Verfremdung und aktivieren darin ein filmisches Potenzial, das Kracauer mit den Worten beschrieben hat, Filme würden ,unsere Umwelt [entfremden], indem sie sie exponieren“. Kracauer: Theorie des Films, S. 105.

64 Vgl. Hermann Kappelhoff: Neue Sachlichkeit. In: Thomas Koebner (Hg.): Reclams Sachlexikon des Films. Stuttgart ${ }^{2} 2007$, S. 470 f. 


\section{Menschen und Dinge}

Durch das Prisma des Querschnittfilms betrachtet, lässt sich aber nicht nur die Anwesenheit präzise gezeichneter Nebenfiguren wie „Koffer-Ede“ (Willi Narloch), dem eifersüchtigen Kfz-Mechaniker (Heinz Scholz) oder dem mürrischen Tankwart (Hans Hardt-Hartloff) bis hinauf zum ehebrecherischen Zahnarzt Dr. Härtel (Willi Schwabe) oder dem blasierten Bühnenstar Günti Schwalbe (Ivan Malré) begründen. Es liefert außerdem einen Ansatzpunkt, um einer weiteren Antriebskraft auf die Spur zu kommen, die in EIN LORD AM ALEXANDERPLATz jenseits herkömmlicher Regeldramaturgien die Ökonomie der Erzählung in Gang hält: das Spiel mit Requisiten, in dem die kleinen und großen Dinge des Lebens sich solange umschmeicheln lassen, bis sie ihre „unbegrenzten Aspekte“ preisgeben. $^{65}$

Vielsagend ist in diesem Zusammenhang, was Erwin Geschonneck in seinen Erinnerungen über die Prämissen seiner Darstellung der Titelfigur schreibt. Abgesehen davon, dass er in dieser Rolle „Eleganz spielen“ konnte, „die immer so ein bißchen schon ,angegangen“ ist, etwas schäbig und angekratzt“, habe es ihm bei der Arbeit an der Figur besonderen Spaß gemacht, „zusammen mit Günter Reisch das Spielen mit Requisiten zu fabulieren“:

Diese Requisiten - steifer Hut, Stock, eine einzelne Rose - sind für Herrn Honig sehr wichtig. Er ist fast nie ohne sie zu sehen. Und wie er mit ihnen spielt, sich in Szene setzt, seinen Charme unterstützt, machte auch einen Großteil seiner Wirkung auf Frauen aus. Noch ein, allerdings sehr großes, Requisit muß ich nennen: ein Auto. [...] Ganz von fern könnte man sich hier daran erinnern, wie Chaplin als Tramp immer mit seinem Stöckchen, seiner Melone und seinen viel zu großen Schuhen spielte. ${ }^{66}$

Man muss den Blickwinkel auf eine später gemachte Äußerung Reischs nur etwas ankippen und die ,Dinge', von denen hier die Rede ist, ganz wörtlich verstehen, um das dazugehörige poetologische Selbstzeugnis des Regisseurs in angemessener Beleuchtung betrachten zu können: „Die Komödie gibt die spielerische Variante zum Verhalten der Menschen in der Realität“, heißt es dort, und weiter:

ich will mit den Dingen und den Möglichkeiten, die in ihnen stecken, jonglieren, sie in Frage stellen, sie aus einer neuen Sicht entdecken können. [...] mein Arbeitsprinzip, sich nach drei

65 Vgl. Kracauer: Theorie des Films, S. 122. Der Wortlaut im Original ist: „Drittens können Filme ein einzelnes Objekt sozusagen lang genug umschmeicheln, um seine unbegrenzten Aspekte zu suggerieren. Da sich auf dieser Route weniger Gelegenheit zu dramatischer Handlung bietet als auf denen räumlicher und kausaler Endlosigkeit, ist sie bisher noch kaum benutzt worden.“ 66 Erwin Geschonneck: Meine unruhigen Jahre. Hg. v. Günter Agde. Berlin 1984, S. 233-234. 
Jahren wieder den heiteren Genres zuzuwenden [...], führt schließlich dazu, daß Dinge sich anreichern, die man mit einem Mal loswerden will. ${ }^{67}$

In EIN LORD AM AlEXANDERPlatz reichert sich die Anwesenheit beweglicher Dinge von Situation zu Situation ganz buchstäblich an, sie werden hervorgeholt und versteckt, deponiert und vergessen, gekauft, verschenkt und entwendet. Als Strukturmotiv durchziehen sie die Handlung, indem sie die einzelnen Situationen miteinander verketten, Interesse und Begehren auf sich ziehen und die Figuren erst eigentlich zueinander ins Verhältnis setzen. ${ }^{68}$ Sie geben prägnanten Aufschluss über verborgene Charaktereigenschaften ihrer Besitzer, wenn etwa in der Werkstatt bei der Durchsicht des Mercedes die Insignien des ehemaligen Diplomatenwagens auf Knopfdruck aus der Stoßstange hervorschnellen. Und sie führen vor Augen, wie schnell sich der äußere Anschein durch Umetikettierung der ,Kennzeichen` verändern lässt. Sie stehen auf diese Weise für ihre Besitzer ein, vor allem Honigs Auto wird konsequent zum dinglichen Alter Ego seines Eigentümers entwickelt. Eine Spiegelkonstruktion von Mensch und Maschine, die sich erst auflöst, als ein sonst nicht weiter in Erscheinung tretender Passant (Winfried Glatzeder) gegen Ende des Films ein weiteres ,Ding“, den als Bremskeil fungierenden Ziegelstein, wegen Eigenbedarfs zum selben Zweck auf einer abschüssigen Straße vom Hinterrad des Mercedes entfernt. Der Mercedes zerschellt in einer Baugrube und wird anschließend stellvertretend für Honigs Junggesellendasein feierlich beigesetzt (Abb. 2.3).

Unentwegt hantieren die Figuren mit Gegenständen, um ihre Absichten und Gefühle zu kommunizieren: Die Gattin des Mechanikers demonstriert ihre Zuneigung zu Honig zunächst einmal damit, dass sie ihm das Hemd wäscht. Im Kosmetiksalon fischt Honig aus seinem Handkoffer voller scheinbar unnützer Dinge im rechten Moment eine Dose Nescafé-Pulver, um die zum Verkauf anstehende Hautcreme noch anziehender zu gestalten, da sie schließlich nicht nur auf, sondern auch unter die Haut der Kundinnen gehen müsse, wie er der staunen-

67 Reisch: Erinnerungen im 30. Jahr der DDR (Teil II), S. 102. Interessant mit Blick auf den spielerischen Anteil ist die an gleicher Stelle von Reisch getroffene Unterscheidung zwischen Komödien, die dramatische Geschlossenheit betonen und denen er lediglich zwei seiner komischen Filme zurechnet (ACH DU FRÖHLICHE... und ANTON DER ZAUBERER), und Lustspielen wie EIN LORD AM ALEXANDERPLATZ, die, wie der Name schon sagt, die Lust am humorvollen Spiel mit dem Sujet in den Vordergrund stellen: „Der Lustspielfilm erlaubt allen Beteiligten, auch dem Zuschauer, das Leben spielerisch und vorwiegend heiter zu betrachten. Die Komödie läßt durch den Spaß so manchen Widerspruch im Ernst des Lebens erkennen und veranlaßt, darüber lachend nachzudenken“ (Reisch: Erinnerungen im 30. Jahr der DDR [Teil II], S. 104).

68 Vgl. Michael Niehaus: Das Buch der wandernden Dinge. Vom Ring des Polykrates bis zum entwendeten Brief. München 2009, S. 31. 

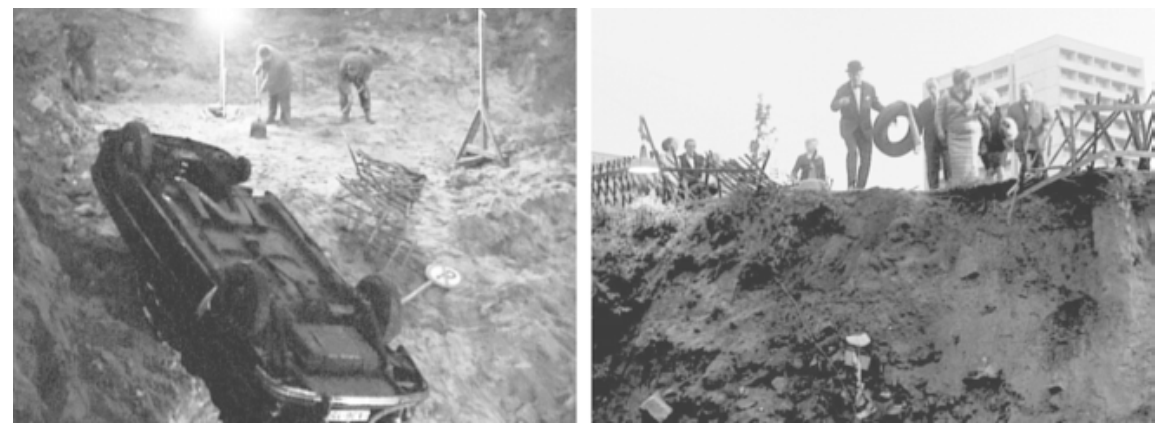

Abb. 2.3: Der Mercedes in der Baugrube.

den Inhaberin des Salons erklärt. Ina, die, wo sie auch geht und steht, unablässig mit den verschiedensten alltäglichen und weniger alltäglichen Gegenständen beschäftigt ist, bringt deren Bedeutung mit der rhetorischen Frage auf den Punkt, was denn ein noch so großes Ruder gegen ein kleines Gaspedal sei. Ein bezeichnendes Kabinettstück führen Vater und Tochter mit der aus dem Westen mitgebrachten Illustrierten Stella auf, die über seine Verurteilung berichtet hatte. Honig will sie schleunigst im Ofen verbrennen, Ina kommt dazwischen, woraufhin die Zeitschrift über mehrere Stationen eine abenteuerliche Reise durch die gesamte Wohnung unternimmt, zu Boden fällt, wieder aufgehoben und hinter dem Rücken versteckt wird, bis sie vorerst in der Küche in einer Kiste landet. Dann jedoch will Honig just am Beispiel dieser Kiste seine ungebrochene Vitalität unter Beweis stellen, indem er sie über seinen Kopf stemmt, wodurch die verfängliche Illustrierte wieder heraus und Ina doch noch in die Hände fällt, die den inkriminierenden Artikel heimlich liest, bevor sie die Zeitschrift schließlich eigenhändig verfeuert.

Die Reihe der Beispiele für die narrative Omnipräsenz beweglicher Gegenstände in EIN LORD AM ALEXANDERPLATz ließe sich beliebig fortsetzen. Von größerer Bedeutung ist, dass über die durch die Hände ihrer Träger und Besitzer quer durch die Stadt wandernden Dinge auch die Brücken geschlagen werden zwischen den verschiedenen Handlungssträngen des Films. Am deutlichsten dort, wo die verschenkten Rosen, Amulette und Haarlocken in der kriminalistischen Untersuchung als Spuren und Indizien gesammelt und konserviert werden, nur um vom Ermittlerpaar Johanna Farkas und Dr. Engelhardt als Fallstricke, in denen sie sich selber verfangen, erneut zum Einsatz zu kommen. Ihren Irrtum erkennen die beiden Ermittler denn auch nur mit Hilfe von Tonbandgerät und Telefon, Küchenmesser und Zigarettenautomat, ohne deren vielfältige Interventionen sie wohl niemals zusammengefunden hätten. 
Parallel zu fassbaren Objekten kursieren im Film Redewendungen und Spruchweisheiten, die als immaterielle Pendants analog zu materiellen Alltagsgegenständen fungieren. ${ }^{69}$ Allen voran die von Honig freigiebig unter seine Mitmenschen verteilte Sentenz, ein Mann solle in seinem Leben einen Baum pflanzen, ein Haus bauen und ein Buch schreiben. In der Liebesszene zwischen Johanna und Dr. Engelhardt erfährt diese Losung ihre finale Umdeutung: Keinen Baum, einen ganzen Wald muss man pflanzen, nicht ein Haus bauen, sondern eine ganze Stadt, und wenn schon ein Buch schreiben, dann jedenfalls nicht über die Psychologie der Frauen. Bündiger lässt sich der Umschlag von bürgerlichchauvinistischen Einzelinteressen an Mitmenschen und Gegenständen nicht in ein Glaubensbekenntnis zur Arbeit am Sozialwohl und Gemeinschaftsgefüge übersetzen. Diesem Credo verdankt sich am Ende auch Honigs moralischer Freispruch, der mit dem Hinweis erfolgt, durch seine - weitgehend im Off des Geschehens erledigten - Versicherungsgeschäfte habe er Kommunalobligationen für das öffentliche Bauwesen zeichnen können, die ihn mit $4 \%$ Zinsen am Bau des Fernsehturms beteiligen.

Es erscheint nur folgerichtig, dass die fast vollzählig vor Gericht versammelten Figuren in der virtuosen Schlussszene des Films, die ihm zugleich als Abspann dient, erstmals von dinglichen Gegenständen weitgehend befreit sind. Als Gleiche unter Gleichen sprechen sie nacheinander ihre Aussage direkt in die Kamera, die damit als letztes, ultimatives ,Objekt‘ am Horizont des Films spürbar wird. Die Einblendung der jeweiligen Darstellernamen hebt die Scharnierfunktion der gewählten filmischen Auflösung noch zusätzlich hervor (Abb. 2.4).

Zuvor kam genau diese Funktion der Off-Stimme des immer schon heterodiegetischen Erzählers zu. Er meldet sich ganz am Schluss noch einmal mit der Bemerkung zu Wort, die Schöpfer des soeben zu Ende gehenden Films „beeilten sich zu versichern“, dass „Ewald Honig vor Rückfällen sicher aus diesem Verfahren entlassen wird“. Wer aber ausgerechnet über einen Versicherungsvertreter etwas „versichern“ zu müssen glaubt, entpuppt sich selbst als Agent von Versicherungen, die ja nichts anderes sind als Wetten auf letztlich noch unabsehbare Wendungen und Ereignisse. Hinter diesem letzten Witz gibt sich auch ein gutes Stück Bescheidenheit im Wirkungskalkül der Filmemacher zu erkennen: Die Quintessenz ihrer Komödie kann am Ende nicht mehr ergeben als die Versicherung einer gelingenden Zukunft. Ob man sich dem romantischen Reiz der Dinge, welche die Großstadt unermüdlich in Gang hält, dauerhaft wird entziehen können, muss sich in der Realität erst erweisen.

69 Vgl. Niehaus: Das Buch der wandernden Dinge, S. 37-39. 

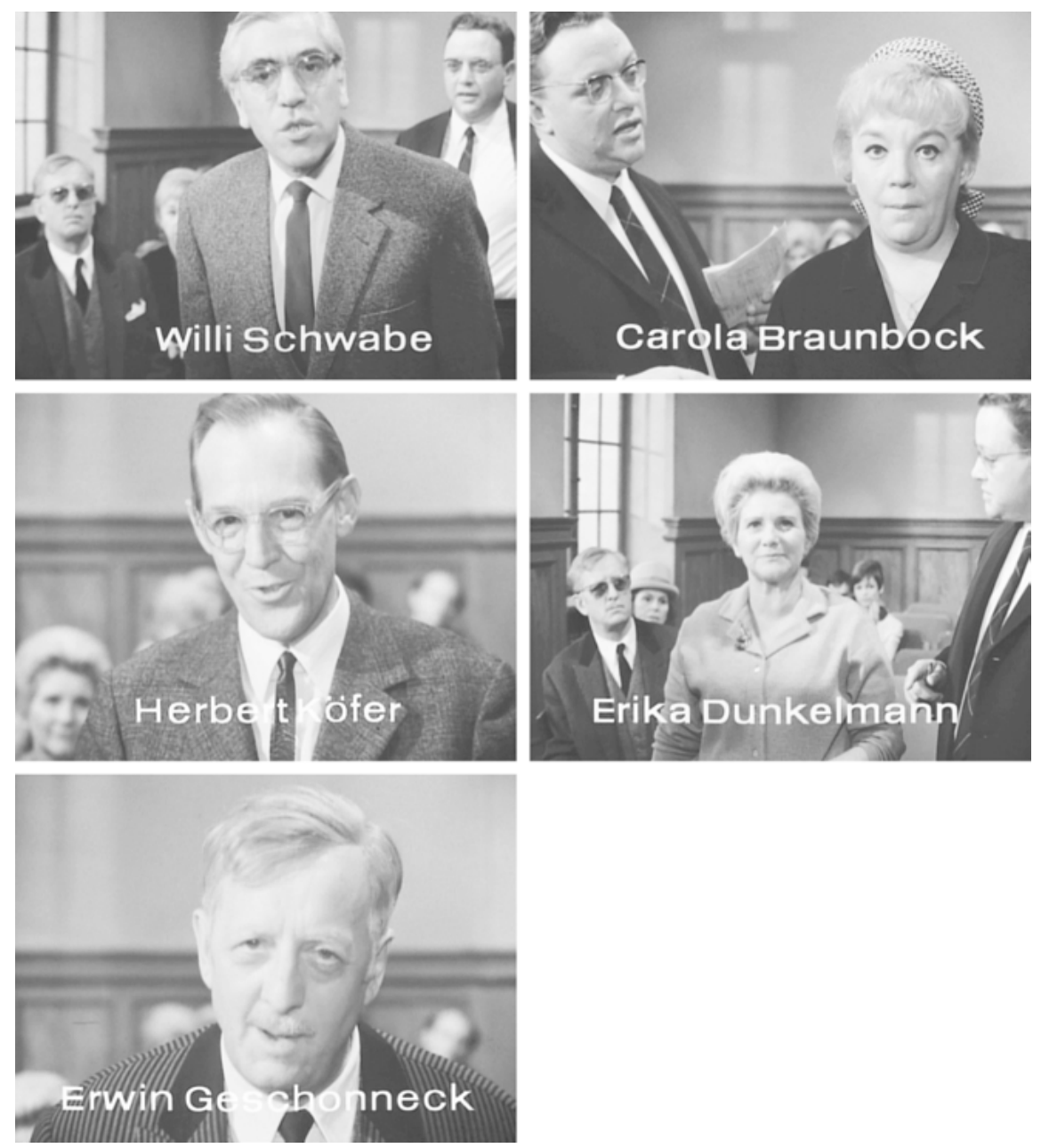

Abb. 2.4: Der Abspann als Aussage. 